\title{
Cardiorenal Syndrome Type 3: Pathophysiologic and Epidemiologic Considerations
}

Sean M. Bagshaw ${ }^{a}$ Eric A. Hoste ${ }^{b} \cdot$ Branko Braam $^{c}$.

Carlo Briguorid • John A. Kellume • Peter A. McCullough ${ }^{f}$. Claudio Ronco 9 for the Acute Dialysis Quality Initiative 11 Working Group

a Division of Critical Care Medicine, Faculty of Medicine and Dentistry, University of Alberta, Edmonton, Alta., Canada; ${ }^{b}$ Department of Critical Care Medicine, University of Gent, Gent, Belgium; 'Division of Nephrology, Departments of Medicine and Physiology, Faculty of Medicine and Dentistry, University of Alberta, Edmonton, Alta., Canada; d Department of Cardiology, Clinica Mediterranea, Naples, Italy; ${ }^{e}$ Clinical Research, Investigation, and Systems Modeling of Acute IIIness Center, Department of Critical Care Medicine, University of Pittsburgh, Pittsburgh, Pa., USA; ${ }^{\text {St. John }}$ Providence Health System, Warren, Mich., Providence Hospitals and Medical Centers, Southfield and Novi, Mich., St. John Macomb Oakland Center, Madison Heights, Mich., St. John Hospital and Medical Center, Detroit, Mich., USA; ${ }^{9}$ Department of Nephrology Dialysis and Transplantation and International Renal Research Institute (IRRIV), San Bortolo Hospital, Vicenza, Italy

\begin{abstract}
Cardiorenal syndrome (CRS) type 3 is a subclassification of the CRS whereby an episode of acute kidney injury (AKI) precipitates and contributes to the development of acute cardiac injury. There is limited understanding of the pathophysiologic mechanisms of how AKI contributes to acute cardiac injury and/or dysfunction. An episode of AKI may have effects that depend on the severity and duration of AKI and that both directly and indirectly predispose to an acute cardiac event. Moreover, baseline susceptibility will modify the subsequent risk for cardiac events associated with AKI. Experimental data suggest cardiac injury may be directly induced by inflammatory mediators, oxidative stress, apoptosis and activation of neuroendocrine systems early after AKI. Likewise, AKI may be associated with physiologic derangements (i.e. volume overload; metabolic acidosis, retention of uremic toxins, hyperkalemia; hypocalcemia), alterations to coronary vasoreactivity, and ventricular remodeling and fibrosis that indirectly exert negative effects on cardiac function. AKI may also adversely impact cardiac function by contributing to alternations in drug pharmacokinetics and pharmacodynamics. Additional experimental and transla-
\end{abstract}


tional investigations coupled with epidemiologic surveys are needed to better explore that pathophysiologic mechanisms underpinning acute cardiac events associated with AKI and their impact on outcomes.

Cardiorenal syndrome (CRS) type 3 (acute renocardiac syndrome) is a subclassification of the CRS whereby an episode of acute kidney injury (AKI) precipitates and contributes to the development of acute cardiac injury and/or dysfunction [1]. There are a number of potential contributing causes for AKI that may predispose to the development of CRS type 3 and which are relevant for the susceptibility, etiology, severity and duration of the type 3 CRS (table 1).

In general, there is limited understanding of the pathophysiologic mechanisms whereby AKI contributes to acute cardiac injury and/or dysfunction. An episode of AKI may have severity and duration-dependent effects that both directly and indirectly predispose to an acute cardiac event. There is some experimental data to suggest that cardiac injury may be directly induced by inflammatory mediators, oxidative stress, and upregulation of neuroendocrine systems early after AKI [2-5]. Likewise, AKI may be associated with physiologic derangements (i.e. volume overload; metabolic acidosis, retention of uremic toxins, hyperkalemia; hypocalcemia), alterations to coronary vasoreactivity, and ventricular remodeling and fibrosis that indirectly exert negative effects on cardiac function [6-10]. The development of AKI may also adversely impact cardiac function, through both direct and indirect mechanisms, by contributing to alternations in drug pharmacokinetics and pharmacodynamics. The relationship between these direct and indirect mechanisms remains poorly understood.

There is a paucity of data describing the epidemiology of acute cardiac events (i.e. arrhythmia, myocardial ischemia, heart failure, cardiac arrest) occurring after an episode of AKI. There is also a lack of data specifically describing the temporal sequence of cardiac events after onset of AKI, including the dose-response effects related to AKI severity and duration on short- and long-term cardiac function $[11,12]$. Moreover, there are challenges when trying to extrapolate on the epidemiology of CRS type 3 from the available literature due to: (1) variability in the methods for defining AKI and heterogeneity in the contributing causes for AKI; (2) variability in the populations at risk being studied and their susceptibility for development of either AKI or acute cardiac events; (3) difficulty in clarifying the temporal association between AKI and acute cardiac events, in particular from observational data, and (4) failure of many observational studies and clinical trials focused on AKI to specifically capture and/or describe the occurrence of acute cardiac events as key outcomes of interest.

Accordingly, the objectives of this review were to: (1) to design a pathophysiologic model of CRS type 3 supported by evidence; (2) identify from this 
Table 1. Summary of potential contributing causes for AKI contributing to CRS type 3

Prototypical condition

Contrast-induced AKI Post-inflammatory GN

Drug-induced AKI Rhabdomyolysis

Major surgery Acute pyelonephritis

Cardiac surgery Post-obstructive uropathy

Table 2. Summary of susceptibilities for CRS type 3

\begin{tabular}{ll}
\hline Risk-modifying factors & \\
Age & Congestive heart failure \\
Sex & Pulmonary disease \\
Coronary artery disease & Chronic kidney disease \\
Hypertension & Systemic vascular disease \\
Hypercholesterolemia & Systemic immune disease \\
Diabetes mellitus & Infection/sepsis \\
\hline
\end{tabular}

model descriptive/epidemiological and mechanistic knowledge gaps, and (3) provide where possible recommendations for future investigation.

\section{Methods}

The 11th Scientific Acute Dialysis Quality Initiative (ADQI) Meeting on Cardio-Renal Syndromes assembled experts on this topic, including cardiologists, nephrologists, intensivists, physiologists and epidemiologists [13].

This report is the result of a modified Delphi analysis performed by the ADQI Working Group [14]. The Delphi method is a structured and standardized process for collecting, summarizing and disseminating knowledge from a group of experts focused on a specific problem or task. A detailed description of the ADQI methodology is available at www.adqi.net [15]. In brief, the process included pre-meeting, at meeting and post-meeting activities.

Prior to the meeting, each member of the working subgroup on the topic of CRS type 3 developed a list of preliminary questions and objectives. The key questions were focused on two broad themes: understanding the pathophysiologic mechanisms and the epidemiology of CRS type 3 (table 2).

Each working group member subsequently performed a systematic literature search focused around these key questions. The literature search was conducted using the Medline database (via the PubMed interface). In general, three broad search themes were used: (1) 'pathophysiology'; (2) 'acute kidney injury' and 'acute renal failure', and (3) 'cardiorenal', 'myocardial infarction', 'heart failure', 'arrhythmia', and 'acute coronary syndrome'. The bibliographies of identified studies and relevant review articles or edito- 
rials were also reviewed along with the personal records of each participating member for additional potentially relevant studies. This literature was reviewed and evaluated to determine the current status of consensus on each of the key questions.

Activities at the meeting, involving the entire working group, included a review of the ADQI methodology, a systematic evaluation of the quality of the retrieved evidence, and incorporation of evidence-based medicine principles into the review process. If evidence on specific key questions was unavailable or lacking, the working subgroup discussed how 'best clinical practice' would be established. The working subgroup used breakout sessions from the main meeting to review, discuss and catalogue the available literature and formulate summary statements to describe existing consensus, best practice and recommendations for future research. The findings from these working subgroup breakout sessions were then presented to the entire ADQI delegate working group at the meeting for feedback and suggested revisions. This method was repeated through an iterative process until majority consensus was achieved.

Post-meeting activities included compilation of the findings and consensus from the meeting and drafting of summary documents.

\section{Findings}

Acute Kidney Injury

AKI is common and increasingly encountered in hospitalized patients. The majority of episodes of severe AKI occur in association with critical illness and are supported in an intensive care setting. Susceptibility to development to AKI likely stems from numerous interacting factors including genetic predisposition [16-19], demographics and burden of comorbid illness such as older age [20], cardiovascular disease, diabetes mellitus and chronic kidney disease (CKD), coupled with clinical events and/or iatrogenic exposures. In addition, the severity and duration of AKI correlated with subsequent risk for complications [21, 22]. However, few data have described the epidemiology of acute cardiac events (i.e. arrhythmia, myocardial ischemia, heart failure, cardiac arrest) temporally following an episode of AKI, including whether dose-response effects related to AKI severity and duration impact short- and long-term risk of cardiac events.

\section{Pathophysiologic Mechanisms of AKI-Induced Cardiac Injury \\ Direct Mechanisms}

AKI is accompanied by almost instantaneous non-hemodynamic events that originate in the kidney and have distant organ effects, including the heart $[2,23]$. The direct physiologic mechanisms by which AKI interacts with the heart have been referred to as the 'cardiorenal connectors' [24]. These mechanisms encompass, but are not likely limited to activation of the systemic immune system (i.e. pro- and anti-inflammatory cytokines and chemokines with alteration in leukocyte trafficking and extravasation); the sympathetic nervous (SNS), renin-angio- 
tensin-aldosterone (RAAS) and coagulation systems; along with increases in oxidative stress (i.e. disruption in the balance between radical oxygen species and nitric oxide (NO)). For each of these 'cardiorenal connector' mechanisms, there is limited experimental evidence to support a pathophysiologic role of AKI directly mediating cardiac events and/or dysfunction.

The majority of experimental studies evaluating the immune 'cardiorenal connectors' have focused on renal ischemia and ischemia/reperfusion injury (IRI). Accordingly, there is little description of the direct interaction of immune response and cardiac performance following AKI in alternative settings (i.e. nephrotoxin, post-obstructive, sepsis). In IRI models, AKI has been shown to elicit a systemic immune response characterized by dose-response increase in circulating levels of pro- and anti-inflammatory mediators. AKI may also contribute to functional alterations in immune cell responsiveness and alterations to leukocyte trafficking, adhesion and tissue extravasation both locally in the kidney and in distal organs such as the heart [2]. Leukocytes have long been thought important in cardiac dysfunction following ischemia and blocking leukocyte function or localization may protect against injury [25]. Elevated white blood cell count is associated with increased risk of acute myocardial infarction [26] and removing white blood cells from cardioplegia solution improves myocardial function in stressed piglet hearts [27].

Several studies have found circulating levels of tumor necrosis factor- $\alpha$ (TNF- $\alpha$ ), interleukin (IL)-1 and IL-6 to increase immediately after renal IRI in experimental models $[3-5,7,28,29]$. Several cytokines have now been implicated as myocardial depressant substances including TNF- $\alpha$, IL-1 $\beta$, IL-6, IL-2, and interferon- $\gamma$ (IFN- $\alpha)$. Elevated TNF- $\alpha$ and IL- 6 have been shown to have direct cardiodepressant effects, characterized by reduced left ventricular (LV) fractional shortening, and elevated LV end-diastolic and systolic dimensions [2, 3, 24, 30]. Cytokines can impact myocardial function due to both impaired myocyte contractility and interaction with the extracellular matrix. In general, cytokine-mediated contractile dysfunction is reversible over an extended time period of several days following exposure [4]. Although these cytokines typically impart negative inotropic effects, the nature and pattern of the inotropic response is more complex, consisting of an immediate response (within minutes) that can be either stimulatory or depressant, depending on the experimental conditions and physiologic milieu, and a delayed response lasting hours to days that is uniformly cardiodepressant and dependent on the production of secondary mediators [4]. The cellular mechanisms underlying these effects are multiple. NO derived from constitutive NO synthase, sphingolipid mediators, arachidonic acid, and alterations in intracellular $\mathrm{Ca}^{2+}$ handling have all been shown to contribute to the immediate contractile effects, whereas the delayed response results primarily from the com- 
bined influence of $\mathrm{NO}$ generated from inducible NOS, the production of radical oxygen species and alterations in $\beta$-adrenergic receptor signaling [4].

In the isolated rat heart, perfusion with TNF- $\alpha$ results in a decrease in LV diastolic pressure and coronary vasoconstriction [31]. Sustained infusion of TNF- $\alpha$ causes time-dependent, delayed ( $2 \mathrm{~h}$ to 2 days) contractile dysfunction and LV dilatation, that could persist for up to 10 days, depending on the dose administered [5]. Reduced LV performance has also been associated with several diastolic abnormalities including slowing of relaxation, LV dilatation without changes in end-diastolic pressure (myocardial creep), and a leftward shift of the end-diastolic pressure-strain relation, indicating increased diastolic stiffness. Pressure-volume studies of the isolated, blood-perfused canine heart have shown that TNF- $\alpha$ acutely impacts LV mechanoenergetics by increasing the oxygen cost of contractility, an effect ascribed to alterations in energy utilization for excitation-contraction coupling [32].

Following IRI, rat hearts also exhibit increased expression of intercellular adhesion molecule-1, myeloperoxidase and apoptosis [3]. Of interest, only renal IRI and not bilateral nephrectomy resulted in a significant increase in myocardial apoptosis, implying systemic inflammation rather than acute uremia may play a more immediate and direct role in myocardial injury and dysfunction in AKI. Moreover, TNF-a likely contributes directly to cardiac myocyte apoptosis, as demonstrated by attenuation of apoptosis following administration of antiTNF- $\alpha$ antibodies [3].

Increased circulating levels of pro-inflammatory cytokines have been demonstrated in patients with chronic heart failure and are associated with maladaptive ventricular remodelling, poor functional status, chronic cachexia and death [33]. Two large randomized trials have evaluated of anti-TNF therapies for atrisk chronic heart failure patients, however while shown to be relatively safe, neither were efficacious for reducing mortality [34]. To date, TNF- $\alpha$ inhibition following an episode of AKI and its effects are myocardial injury and/or function have not yet been evaluated. By extrapolation, this concept may have merit for CRS type 3, in settings where moderate to severe AKI induces a strong immune response characterized by markedly elevated levels of inflammatory mediators, in particular those with direct myocardial effects such as TNF- $\alpha$. As an example, in a folic-acid-induced rat model of AKI, administration of a single low dose of cyclosporine A ( $1-5 \mathrm{mg} / \mathrm{kg})$ not only markedly attenuated development of AKI (i.e. rise in serum creatinine, urea) but also attenuated systemic IL-6 expression, TNF-like weak inducer of apoptosis (TWEAK) expression, nuclear factor- $\kappa B$ activation, inflammatory cell infiltration and renal tubular cell apoptosis when compared with controls [35]. Recently, deficiency of heme oxygenase-1, an enzyme induced by oxidative stress and responsible for degradation of heme mol- 
ecules, was found to exacerbate induction of systemic IL-6 along with IL-6 mRNA and downstream signaling effectors in myocardial tissue [28].

Alternatively, milder forms of renal IRI may have a 'protective' effect on the heart against ischemic injury. In a rat study of brief $(15 \mathrm{~min})$ renal artery occlusion under hypothermic conditions $\left(30^{\circ} \mathrm{C}\right)$, Gho et al. [36] found marked reductions in myocardium at risk and total area infarction when compared with controls, however renal IRI injury was failed to attenuate injury at normothermia. The precise mechanisms by which mild renal IRI may protect against downstream myocardial ischemia are incompletely understood, however may in part be mediated by alterations in sympathetic outflow coupled with upregulation of anti-inflammatory mediators and anti-apoptotic responses [36, 37].

There is limited data available describing the role of neuroendocrine activation, specifically the SNS and RAAS in the pathophysiologic of CRS type 3. However, activation of the SNS is a hallmark of both AKI and acute HF [38]. The enhancement of renal SNS activity and its consequent effect on norepinephrine overflow from nerve terminals during AKI [39] may impair myocardial function through several mechanisms, including: (1) direct effects of norepinephrine, (2) disturbances in myocardial $\mathrm{Ca}^{2+}$ homeostasis, (3) increase in myocardial oxygen demand contributing to susceptibility to endocardial ischemia, (4) cardiac myocyte apoptosis mediated through $\beta_{1}$-adrenergic receptors stimulation [40], (5) stimulation of $\alpha_{1}$-adrenergic receptors, contributing to cardiac myocyte hypertrophy, and (6) direct activation of the RAAS. Heightened adrenergic drive can stimulate $\beta_{1^{-}}$ adrenergic receptors in the juxtaglomerular apparatus of the kidneys contributing to reduced renal blood flow and exacerbated release of renin and RAAS activation. Maladaptive RAAS activation in AKI contributes to angiotensin II (ANGII) release, vasoconstriction and further loss of extracellular fluid homeostasis. ANGII may redundantly contribute to systemic vasoconstrictor and excessive elevation of the systemic vascular resistance. ANGII may also play a direct role in modifying myocardial structure and function [41], contribute to cellular hypertrophy, and precipitate apoptosis in cardiac myocyte cultures [42]. ANGII is also a potent stimulator of a number of cell signaling pathways including those involved in oxidative stress, inflammation, and the regulation of the extracellular matrix [43].

In a dog model of renal IRI, Kingma et al. [8] implied increased activity of the RAAS contributing to observed diminished coronary responsiveness to acetylcholine, adenosine, bradykinin, and L-arginine. In addition, renal IRI resulted in greater myocardial oxygen consumption at rest. While a definitive role for the RAAS was not established, these data imply that AKI may directly contribute to impaired coronary vasoreactivity and elevated myocardial oxygen consumption that would potentially increase susceptibility to myocardial ischemia and major cardiac events. Several rat models of renal IRI have shown increased 
secondary lung injury due largely to alternation in pulmonary vascular permeability with resultant interstitial edema and alveolar hemorrhage [2]. The altered pulmonary vascular permeability associated with AKI is directly mediated by systemic inflammatory mediators and kidney-lung cross-talk. The resulting alveolar congestion and edema is in part mediated by altered expression of epithelial sodium channel and aquiporin-5 expression. Similar mechanisms may also contribute to myocardial interstitial edema and dysfunction [44]. Transgenic sickle mice subjected to renal IRI exhibited significantly increased myocardial capillary congestion, serum amyloid-P (murine equivalent of C-reaction protein), and renal expression of caspase-3 compared with controls [45].

In addition, while we considered the cardiac-specific cellular responses associated with AKI including the role of mitochondrial dysfunction, apoptosis, remodelling, and fibrosis, limited data were available. Robinson et al. [46] evaluated cardiac mitochondrial function in a rat model of glycerol-induced AKI. In this study, isolated heart preparations showed site-unspecific uncoupling of oxidative phosphorylation. This contributed to increased mitochondrial $\mathrm{Ca}^{2+}$ uptake, and may provide explanation for the diminished inotropic response in the setting of AKI.

Cardiac myocyte apoptosis and neutrophil infiltration are important contributors to the pathophysiology of myocardial infarction during AKI and transgenic models have shown that even apoptosis alone can lead to lethal heart failure [3]. In this study, kidney IRI, but not uremia, was critical to induction of apoptosis in myocardial tissue. Another experimental model of cisplatininduced AKI found an increased number of TUNEL-positive cells implying significant myocardial apoptosis [47]. It has been demonstrated that apoptotic rates of only 23 cardiac myocytes per $10^{5}$ nuclei are sufficient to induce a lethal, dilated cardiomyopathy [48]. Although this frequency of cell death is quite low, it is still 15-fold higher than that observed in healthy human hearts. Decreasing intracellular iron levels is also known to induce apoptosis [49].

The strongest evidence of a cardiorenal link between AKI and the development of cardiac fibrosis is the $\beta$-galactoside-binding lectin galectin-3 [9, 10]. Galectin-3 mRNA expression in renal tubules was shown to be upregulated early after IRI and toxin-induced AKI and persisted for 7 days following injury [9]. Importantly, galectin-3 has been implicated in the development of myocardial fibrosis and heart failure in an experimental model of AKI [10]. Moreover, inhibition of galectin-3 can mitigate the formation of cardiac fibrosis [50].

\section{Indirect Mechanisms}

The workgroup identified a number of potential consequences of AKI that may act to indirectly contribute to reduced cardiac function (fig. 1, 2). 


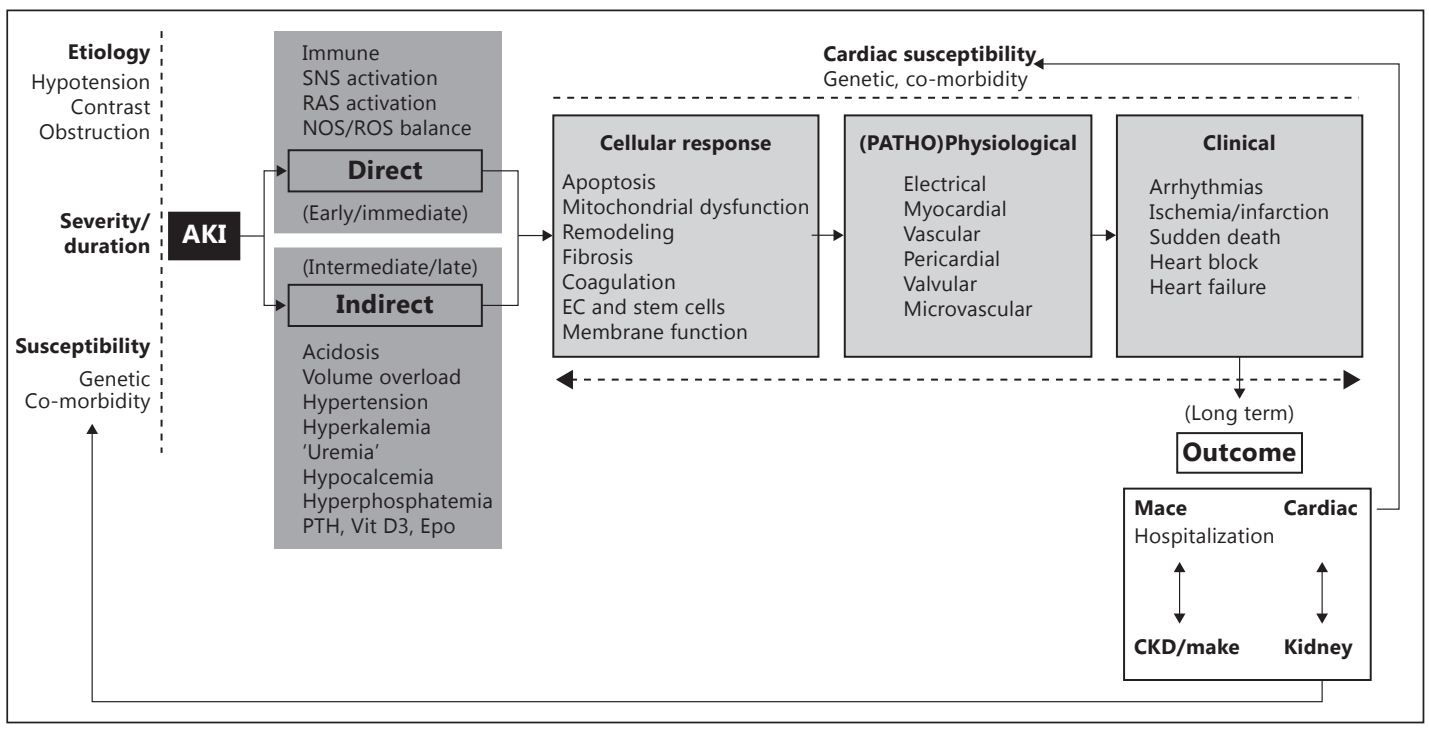

Fig. 1. Summary of the demographic contributors, clinical susceptibilities and pathophysiologic mechanisms for development of CRS type 3. Reproduced with permission from ADQI [88].

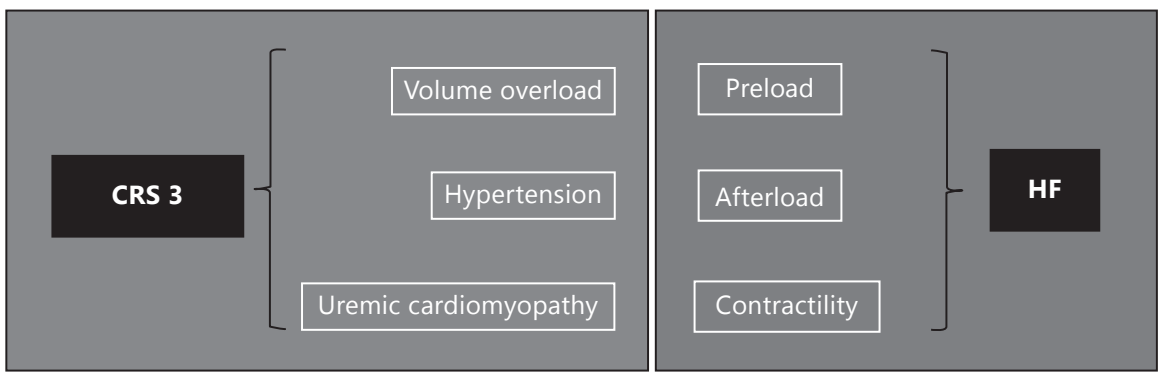

Fig. 2. Summary of the interaction of clinical complications of AKI and theoretical negative physiologic effects on myocardial performance. Reproduced with permission from ADQI [88].

Volume Overload. Excess fluid accumulation is associated with less favorable outcomes in critically ill patients. In a large randomized trial of fluid management strategies in critically ill patients with acute lung injury, a conservative fluid regimen was associated with improved lung function and a trend for lower utilization of renal replacement therapy (RRT) compared with a liberal fluid regimen [51]. Similar data have also been shown in critically ill children [52]. Moreover, excess fluid accumulation may worsen kidney function through venous congestion and 
intra-abdominal hypertension. Several observational studies, while not established causality, consistently show association between fluid overload and less favorable outcomes in critically ill patients with AKI $[53,54]$. In addition, fluid removal in these patients, either through loop diuretics use [55] or ultrafiltration [56], was associated with higher survival. Fluid accumulation may adversely impact myocardial performance [44], predispose to ventricular arrhythmias [57] and may contribute to maladaptive ventricular remodelling and myocardial fibrosis $[44,58]$.

Hypertension. The majority of critically ill patients with AKI may present with hypotension due to the precipitating etiology for AKI (i.e. major surgery, sepsis, cardiogenic shock), however beyond the inciting event contributing to CRS type 3, a number of mechanisms may contribute to the development of both systemic and pulmonary hypertension. This may occur through volume overload contributing to elevated preload, alterations in cardiac output and increased afterload through total body autoregulation, activation of the SNS and RAAS. In susceptible patients, these may contribute to myocardial ischemia, arrhythmias and ventricular dysfunction. Increased activation of the SNS and RAAS has been well described in conditions such as hepatorenal syndrome and acute decompensated heart failure [59].

Acidemia. Metabolic acidosis is a common complication of AKI. In a recent survey of critical care clinicians, $74 \%$ of the respondents stated that metabolic acidosis was a key factor contributing to a decision to initiate RRT among critically ill patients [60]. In the RENAL study, a large prospective randomized study evaluating the intensity of continuous RRT (CRRT), 35\% of critically ill patients had severe acidemia at time of initiation of CRRT [61]. The accumulated acid (i.e. $\mathrm{H}^{+}$ions) may alter the protein structure and interfere with normal function. This may directly contribute to decreased myocardial contractility through changes to $\beta$-receptor expression and altered intracellular calcium handling [62].

Hyperkalemia. Similar to acid-base homeostasis, potassium is predominantly dependent on kidney function for elimination from the body. Hyperkalemia is a life-threatening complication of AKI and an important absolute indication for RRT [60], however studies of patients with severe AKI show hyperkalemia is a relatively infrequent reason for RRT initiation [63]. Hyperkalemia is associated with cardiac toxicity and arrhythmic complications (see below).

Hypophosphatemia. While untreated AKI is often associated with elevated plasma phosphate levels, patients started on RRT commonly develop hypophosphatemia [61, 64, 65]. Hypophosphatemia may be an unrecognized iatrogenic complication associated with respiratory muscle weakness and impaired myocardial performance $[66,67]$.

Uremic Toxins. In CKD, more than 100 uremic toxins or retention products have been identified [68]. Uremic toxins include water-soluble, protein-bound 
and small molecular weight compounds with variable pharmacodynamic profiles. Several uremic toxins (i.e. indoxylsulfate, $p$-cresol conjugates, $\beta_{2^{-}}$ microgliobulin, and FGF-23) are associated with cardiovascular toxicity in CKD [69]. However, there is a relative paucity of data on acute uremia in AKI. In AKI, uremic compounds may exert different time-dependent physiologic effects when compared to those described in CKD. There is indirect evidence to suggest uremic compounds in AKI may negatively impact kidney function. For example, coronary vasoreactivity is adversely affected in AKI, resulting in altered thresholds for myocardial ischemia in conditions of increased myocardial oxygen demand [8]. Uremic toxicity is complicated, as varying duration and severity of AKI as well as concomitant non-renal organ dysfunctions may synergistically act to adversely impact cardiac function.

\section{Pathophysiological Effects of AKI on the Heart}

The pathophysiologic mechanisms underlying the interplay between kidney injury and heart dysfunction are still not completely understood (fig. 2). However, we can summarize several of the physiologic effects whereby AKI may negatively impact heart function as: (a) electrophysiological, (b) ischemic, (c) myocardial and/or (d) pericardial.

\section{Electrophysiological Effects}

The loss of acid-base, electrolyte and fluid homeostasis occurring in AKI may precipitate alteration in heart rhythm. Electrical signaling in the heart involves the passage of ions through ionic channels. The $\mathrm{Na}^{+}, \mathrm{K}^{+}, \mathrm{Ca}^{2+}$, and $\mathrm{Cl}^{-}$ions are the major charge carriers, and their movement across the cell membrane creates a flow of current that generates excitation and signals contraction in cardiac myocytes.

Hyperkalemia is associated with a distinctive sequence of electrocardiogram (ECG) changes. The earliest is usually narrowing and peaking (tenting) of the $\mathrm{T}$ wave (fig. 3). The ratio of intracellular to extracellular potassium is important for determining the cellular membrane potential. Small changes in the extracellular potassium level can have profound effects on the function of the electrical conduction system of the heart. In the setting of hyperkalemia, the excess extracellular potassium will increase the activity of the potassium channel, resulting in faster repolarization and thus creating the narrow base and tall $\mathrm{T}$ wave. On the other hand, this will inactivate the sodium channel, preventing proper conduction of electrical activity. Progressive extracellular hyperkalemia reduces atrial and ventricular resting membrane potentials, thereby inactivating sodium channels. Moderate to severe hyperkalemia occasionally induces ST segment elevations in the right precordial leads (V1 and V2) and simulates an ischemic 

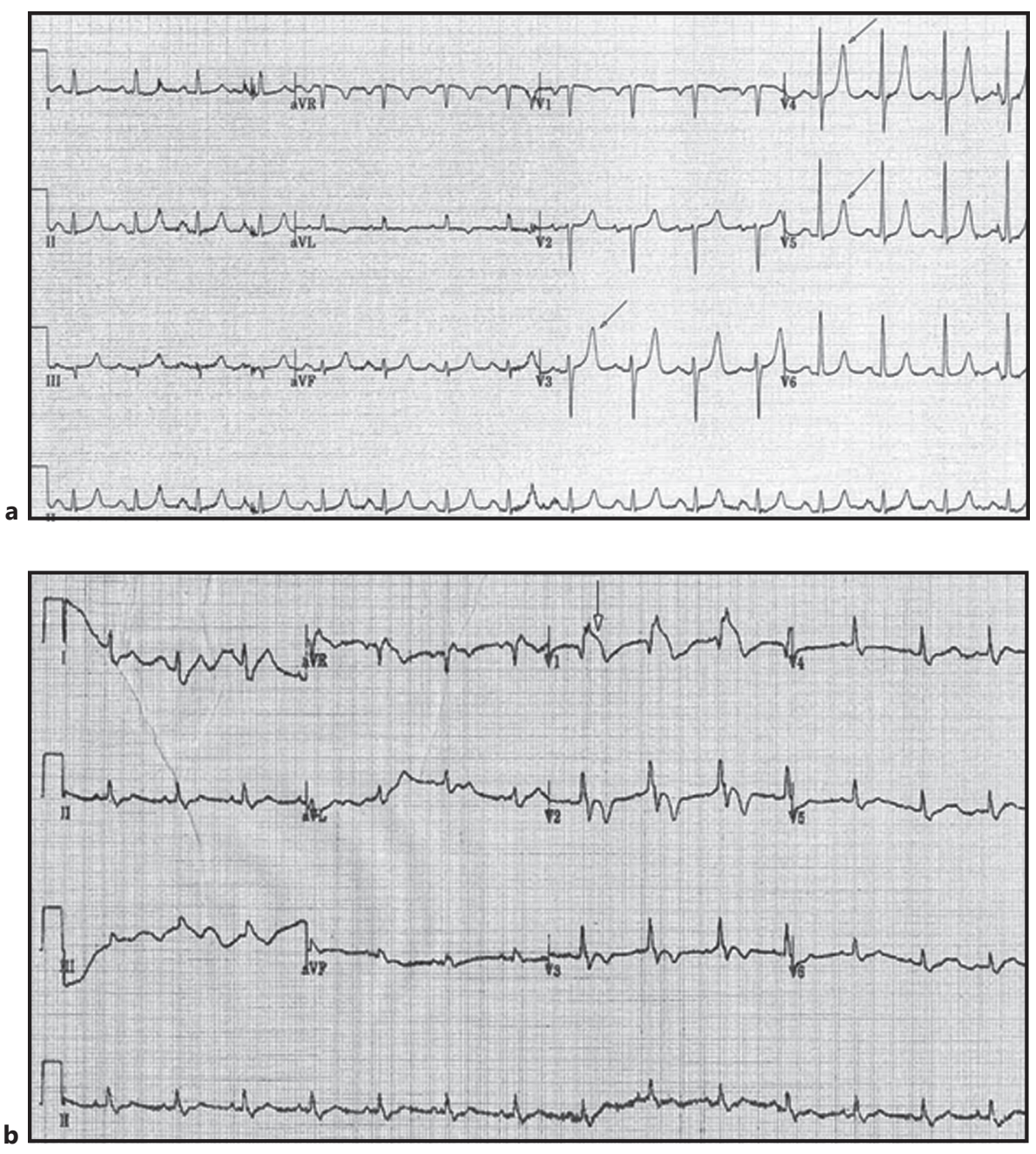

Fig. 3. Hyperkalemia. a A 54-year-old woman with acute renal failure due to glomerulonephritis status was admitted with acute onset of shortness of breath. Her clinical examination and radiographic findings were consistent with acute pulmonary edema. ECG shows normal sinus rhythm with tall T waves best seen in leads V3, V4 and V5. The tall T wave is symmetrical, narrow, scooped inwards and 'tented'. $\mathrm{K}^{+}$level was $5.8 \mathrm{mEq} / \mathrm{l}$. This classic appearance of the T wave is usually associated with hyperkalemia. The PR interval, QRS duration and QTc interval are all within normal range. The $P$ wave is wide $(>0.12 \mathrm{~s}$ in duration) and bifid in shape - so-called 'P-mitrale' in lead II. There is a negative component in lead V1, which is widened and slurred, consistent with left atrial enlargement. b ECG shows significant widening of the QRS complex to $158 \mathrm{~ms}$ with right bundle branch block morphology associated with coved ST segment elevation in lead V1, resembling 'Brugada ECG pattern', which can be seen in severe hyperkalemia. Reproduced with permission from ADQI [88]. 


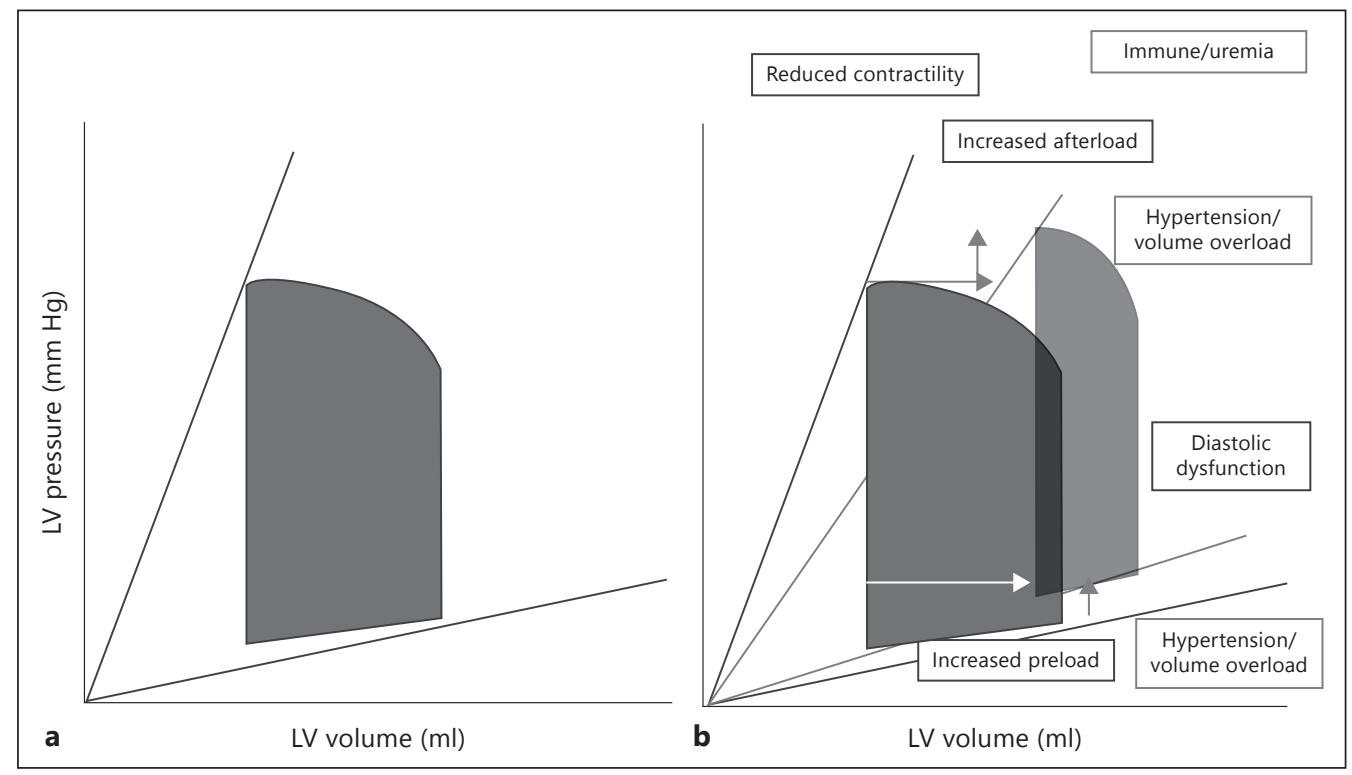

Fig. 4. a, b Mechanisms of LV dysfunction in CRS type 3. Reproduced with permission from ADQI [88].

current-to-injury pattern. However, even severe hyperkalemia can be associated with atypical or non-diagnostic ECG findings. Hyperkalemia can induce a Brugada-like pattern in the ECG [70]. This usually occurs in critically ill patient with significant hyperkalemia (serum $\left[\mathrm{K}^{+}\right]>7.0 \mathrm{mmol} / \mathrm{l}$ ) and is associated with pseudo-right bundle branch block and persistent ST segment elevation in at least two precordial leads (fig. 4). Very marked hyperkalemia leads to eventual asystole, sometimes preceded by a slow undulatory (sine-wave) ventricular flutter-like pattern [71].

Severe hypermagnesemia can cause AV nodal and intraventricular conduction disturbances that may culminate in complete heart block and cardiac arrest.

An increased extracellular calcium concentration shortens the ventricular action potential duration by shortening phase 2 of the action potential. This cellular change correlates with the shortening of the QT interval (ST segment portion). Hypercalcemia may produce a high takeoff of the ST segment in leads V1 and V2 that can simulate acute ischemia. $\mathrm{Ca}^{2+}$ plays a central role in the regulation of myocardial contraction and relaxation and there is increasing evidence that disturbances in $\mathrm{Ca}^{2+}$ handling may play a central role in the disturbed contractile function in heart failure. The intracellular $\mathrm{Ca}^{2+}$ transient demonstrates a blunted rise with depolarization, reflecting slower delivery of $\mathrm{Ca}^{2+}$ to the con- 
tractile apparatus (causing slower activation) and a slower rate of fall during repolarization (causing slower relaxation). These two abnormalities could explain both systolic and diastolic dysfunction [72].

\section{Ischemic Effects}

The theoretical pathophysiological mechanisms responsible of myocardial ischemia during AKI may also include the following: (1) acidemia; (2) neurohormonal activation (i.e. SNS and RAAS), and (3) acute accumulation of uremic toxins and cytokines [31]. The SNS activation can lead to vasoconstriction and increased myocardial oxygen demand. These can lead to ischemia when oxygen delivery is restricted, in particular in susceptible patients. TNF- $\alpha$ induces coronary vasoconstriction, which contributes indirectly to contractile dysfunction in isolated, crystalloid-perfused rat hearts [73]. Endothelin and bradykinin may be also responsible for the reduced vessel function. Endothelin is a potent peptide vasoconstrictor released by endothelial cells throughout the circulation. Its release from endothelial cells can be enhanced by several vasoactive agents (ANGII, norepinephrine) and cytokines (TNF, and IL-1 $\beta$ ). During AKI, higher levels of myocardial oxygen demand would trigger maldistribution of ventricular blood flow and result in subendocardial ischemia. It has been demonstrated that during AKI (1) coronary autoregulation is preserved but the lower autoregulatory break point shifts to a higher coronary perfusion pressure; (2) coronary vascular reserve and coronary vascular conductance are markedly diminished, and (3) coronary vessel reactivity to either endothelium-dependent or -independent vasodilators is substantially reduced [8]. The observed reduction in coronary venous $\mathrm{PO}_{2}$ during AKI may reflect: (1) a reduced bioavailability of $\mathrm{NO}$ to vascular smooth muscle cells as a result of structural alterations in the vessel or microvessel wall, (2) an increased destruction of $\mathrm{NO}$ as a result of overproduction of reactive oxygen intermediates in the vessel wall, and (3) an impaired dilatation to NO donors (e.g. nitroglycerin) as a result of defective vascular smooth muscle cell function at the level of the soluble guanylate cyclase/cyclic guanosine monophosphate signaling pathway [74].

Recent evidence has emerged to implicate a potentially important pathophysiologic link between neutrophil gelatinase-associated lipocalin (NGAL), a $25-\mathrm{kDa}$ lipocalin covalently bound to gelatinase from human neutrophils involved inflammation and cellular matrix degradation, and CRSs [75]. NGAL is a strong marker of acute renal tubular injury [76]. Renal NGAL expression rapidly increased following acute inflammation and/or injured renal tubular epithelia, in particular following damage from IRI and toxin exposure [77]. NGAL is also believed to play an important role in iron homeostasis and traffic and may activate apoptotic pathways by regulating the intracellular iron concentration 
[78]. NGAL has also been implicated in the pathophysiology of heart failure, myocarditis and in coronary atherosclerosis [79-81]. Likewise, urinary kidney injury molecule-1, a specific marker of renal tubular damage, has also been implicated for modifying the susceptibility to heart failure risk [82]. These data suggest that acutely upregulated biomarkers of kidney damage, such as NGAL and kidney injury molecule-1, may circulate to have distant pathophysiologic effects on the heart, including myocardial inflammation, destabilization of atherosclerotic plaques and susceptibility to ischemic events.

\section{Myocardial Effects}

In animal experiments, it has been shown that $48 \mathrm{~h}$ after kidney IRI, functional changes in the myocardium including LV dilatation, increased LV end-diastolic and end-systolic diameters, increased relaxation time, and decreased fractional shortening are evident [3]. The proposed pathophysiological mechanisms responsible for LV dysfunction following an episode of AKI include accumulation of inflammatory mediators (i.e. cytokines); leukocyte infiltration, SNS activation; acute sodium and fluid retention; metabolic acidosis, and accumulation of uremic toxins.

The ventricular pressure-volume loop provides a paradigm to understand the relationship between intraventricular pressure and volume throughout the cardiac cycle. Figure 4 shows the pressure-volume relationship under normal conditions and following development of severe AKI. The combined global effect was therefore postulated. The theoretical detrimental effects of AKI on myocardial mechanics may include: (1) increase in preload due to intravascular fluid accumulation (causes a rightward shift of the LV loop); (2) increase in afterload due to increased mean systemic blood pressure (causes an increase in LV end-systolic pressure); (3) increase in diastolic dysfunction due to loss of calcium homeostasis (causes an upward shift of the LV loop), and (4) increase in systolic dysfunction due to inflammatory mediators and uremic toxins (causes a reduction in contractility).

\section{Pericardial Effects}

Uremia through the myocardial-depressant factors (cytokines) may precipitate pericarditis [83].

\section{Clinical Outcomes Associated with CRS Type 3}

There is limited data in the literature on the impact of AKI on occurrence of cardiac-specific events and outcomes (table 3). However, in a large populationbased cohort study, James et al. [84] evaluated the association of AKI following coronary angiography (i.e. CI-AKI) and occurrence of cardiovascular-related 
Table 3. Summary of key questions proposed by the ADQI Working Group

Pathophysiologic mechanisms of CRS type 3

1. What are the most important 'direct' pathophysiologic mechanisms following an episode of AKI that contribute to cardiac injury/dysfunction?

a. What is the time course (temporality)?

b. What is the impact of etiology, severity and/or duration (dose response)?

2. What are the most important 'indirect' pathophysiologic mechanisms following an episode of AKI that contribute to cardiac injury/dysfunction?

a. What is the time course (temporality)?

b. What is the impact of etiology, severity and/or duration?

3. What is the relationship between direct and indirect pathophysiologic mechanisms on cardiac injury/dysfunction?

a. What is the relationship between cardiac pressure-volume mechanics (i.e. indirect) and myocardial function (i.e. direct) following an episode of AKI?

Epidemiology of CRS type 3

1. What are the important factors modifying the risk/susceptibility for cardiac-specific events after an episode of AKI?

a. Baseline susceptibilities kidney and cardiac-specific factors (non-modifiable)?

2. What are the key contributing factors for development of AKI in CRS type 3?

a. What is an ideal representative etiology for AKI to describe the CRS type 3 (i.e. contrast-induced nephropathy)

b. What is the role iatrogenesis in CRS type 3?

3. What is the incidence and spectrum of cardiac-specific events following an episode of AKI?

4. What is the time course for cardiac-specific events following an episode of AKI? (ESC guidelines $\sim 1$ month)

5. What are the implications of CRS type 3 on the natural history of cardiac function?

hospitalizations, in addition to mortality and end-stage kidney disease (ESKD). Increasing severity of CI-AKI was associated with a dose-response increase in risk of heart failure hospitalization (AKIN stage I: HR 1.48, 95\% CI 1.16-1.91; AKIN stage II/III: 2.17, 95\% CI 1.49-3.15). Similar patterns were also seen for mortality, progression to ESKD and AKI-related hospitalization. Anzai et al. [12] prospectively evaluated 141 consecutive patients receiving reperfusion therapy after presentation with first anterior ST-elevation myocardial infarction, of which 22.0\% ( $\mathrm{n}=31)$ developed AKI within $48 \mathrm{~h}$, defined as an increase in serum creatinine $>0.3 \mathrm{mg} / \mathrm{dl}(26.5 \mu \mathrm{mol} / \mathrm{l})$. The development of early post-reperfusion AKI was independently associated with higher risk for in-hospital cardiac events including heart failure, ventricular arrhythmia, and cardiac-specific death, along with long-term cardiac events including rehospitalization for heart failure and major adverse cardiac events (MACE). Cardiovascular-related deaths are the second most common cause of death after sepsis, predominantly attributable to heart failure and ischemic cardiac events, in patients with AKI [85]. 
Table 4. Summary of cardiac-specific events

Spectrum of outcome events

Heart failure

Myocardial infarction

Ventricular arrhythmia

Major adverse cardiac event (MACE)

Accelerated loss cardiac function

Cardiac surgery

Mechanical support device

Death

Hospitalization

\section{Consensus Recommendations for Future Research}

Future investigations focused on CRS type 3 should surveillance for cardiacspecific events (table 4) not only during the in-hospital period but also during long-term follow-up (i.e. 6-12 months). CRS type 3 may be associated with both downstream cardiac and kidney-specific adverse outcomes. In addition to cardiac-specific events, the occurrence of kidney-specific outcomes, including subsequent episodes of AKI [86], incident CKD, progression to ESKD and MACE should be captured. Future investigations should also integrate an assessment of baseline susceptibility for cardiac-specific events following development of CRS type 3 (i.e. low, intermediate, high) and evaluate how these susceptibilities are modified by either the severity of AKI and the duration of AKI. For example, risk of a cardiac-specific event associated with AKI may be similar for a patient suffering a mild but extended episode of AKI compared with a patient developing a more severe episode of relative short duration.

\section{Consensus Recommendations for Clinical Practice}

Evolving data suggest that surrogate measures of kidney-related damage, such as hypertension and proteinuria, may represent simply screening measures to predict long-term decrements in kidney function [87].

\section{Conclusions}

CRS type 3 represents a poorly understood subgroup of heart-kidney interaction, both with respect to the precise pathophysiologic mechanisms and its epidemiology. Additional experimental and translational investigations coupled with epidemiologic surveys are needed to better explore that pathophysiologic mechanisms underpinning acute cardiac events associated with AKI and their impact on outcomes. 


\section{References}

1 Ronco C, Haapio M, House AA, Anavekar N Bellomo R: Cardiorenal syndrome. J Am Coll Cardiol 2008;52:1527-1539.

2 Yap SC, Lee HT: Acute kidney injury and extrarenal organ dysfunction: new concepts and experimental evidence. Anesthesiology 2012;116:1139-1148.

3 Kelly KJ: Distant effects of experimental renal ischemia/reperfusion injury. J Am Soc Nephrol 2003;14:1549-1558.

4 Prabhu SD: Cytokine-induced modulation of cardiac function. Circ Res 2004;95:11401153.

5 Bozkurt B, Kribbs SB, Clubb FJ Jr, et al: Pathophysiologically relevant concentrations of tumor necrosis factor- $\alpha$ promote progressive left ventricular dysfunction and remodeling in rats. Circulation 1998;97: 1382-1391.

6 Zager RA: Uremia induces proximal tubular cytoresistance and heme oxygenase-1 expression in the absence of acute kidney injury. Am J Physiol Renal Physiol 2009; 296:F362-F368.

7 Zager RA, Johnson AC, Lund S: Uremia impacts renal inflammatory cytokine gene expression in the setting of experimental acute kidney injury. Am J Physiol Renal Physiol 2009;297:F961-F970.

-8 Kingma JG Jr, Vincent C, Rouleau JR, Kingma I: Influence of acute renal failure on coronary vasoregulation in dogs. J Am Soc Nephrol 2006; 17:1316-1324.

$\checkmark 9$ Nishiyama J, Kobayashi S, Ishida A, et al: Upregulation of galectin- 3 in acute renal failure of the rat. Am J Pathol 2000;157:815-823.

10 Sharma UC, Pokharel S, van Brakel TJ, et al: Galectin-3 marks activated macrophages in failure-prone hypertrophied hearts and contributes to cardiac dysfunction. Circulation 2004;110:3121-3128.

$\checkmark 11$ Chuasuwan A, Kellum JA: Cardio-renal syndrome type 3: epidemiology, pathophysiology, and treatment. Semin Nephrol 2012;32:31-39.

12 Anzai A, Anzai T, Naito K, et al: Prognostic significance of acute kidney injury after reperfused ST-elevation myocardial infarction: synergistic acceleration of renal dysfunction and left ventricular remodeling. J Card Fail 2010;16:381-389.
13 Ronco C, McCullough P, Anker SD, et al: Cardio-renal syndromes: report from the consensus conference of the Acute Dialysis Quality Initiative. Eur Heart J 2010;31:703711.

14 Ronco C, Kellum JA, Mehta R: Acute Dialysis Quality Initiative (ADQI). Nephrol Dial Transplant 2001;16:1555-1558.

15 Kellum JA, Bellomo R, Ronco C: Acute Dialysis Quality Initiative (ADQI): methodology. Int J Artif Organs 2008;31:9093.

16 Perianayagam MC, Tighiouart H, Liangos O, et al: Polymorphisms in the myeloperoxidase gene locus are associated with acute kidney injury-related outcomes. Kidney Int 2012;82: 909-919.

17 Payen D, Lukaszewicz AC, Legrand M, et al: A multicentre study of acute kidney injury in severe sepsis and septic shock: association with inflammatory phenotype and HLA genotype. PLoS One 2012;7:e35838.

18 Frank AJ, Sheu CC, Zhao Y, et al: BCL2 genetic variants are associated with acute kidney injury in septic shock. Crit Care Med 2012;40:2116-2123.

19 Lu JC, Coca SG, Patel UD, Cantley L, Parikh CR: Searching for genes that matter in acute kidney injury: a systematic review. Clin J Am Soc Nephrol 2009;4:1020-1031.

20 Coca SG, Cho KC, Hsu CY: Acute kidney injury in the elderly: predisposition to chronic kidney disease and vice versa. Nephron Clin Pract 2011;119(suppl 1):c19-c24.

21 Brown JR, Kramer RS, Coca SG, Parikh CR: Duration of acute kidney injury impacts long-term survival after cardiac surgery. Ann Thorac Surg 2010;90:1142-1148.

22 Chawla LS, Amdur RL, Amodeo S, Kimmel PL, Palant CE: The severity of acute kidney injury predicts progression to chronic kidney disease. Kidney Int 2011;79:1361-1369.

23 Grams ME, Rabb H: The distant organ effects of acute kidney injury. Kidney Int 2012;81: 942-948.

24 Bongartz LG, Cramer MJ, Doevendans PA, Joles JA, Braam B: The severe cardiorenal syndrome: 'Guyton revisited'. Eur Heart J 2005;26:11-17. 
25 Ma XL, Lefer DJ, Lefer AM, Rothlein R: Coronary endothelial and cardiac protective effects of a monoclonal antibody to intercellular adhesion molecule-1 in myocardial ischemia and reperfusion. Circulation 1992;86:937-946.

26 Ernst E, Hammerschmidt DE, Bagge U, Matrai A, Dormandy JA: Leukocytes and the risk of ischemic diseases. JAMA 1987;257: 2318-2324.

27 Kronon MT, Allen BS, Halldorsson A, Rahman S, Wang T, Ilbawi M: Dose dependency of $\mathrm{L}$-arginine in neonatal myocardial protection: the nitric oxide paradox. J Thorac Cardiovasc Surg 1999;118: 655-664.

28 Tracz MJ, Juncos JP, Croatt AJ, et al: Deficiency of heme oxygenase-1 impairs renal hemodynamics and exaggerates systemic inflammatory responses to renal ischemia. Kidney Int 2007;72:1073-1080.

29 Hirota H, Yoshida K, Kishimoto T, Taga T: Continuous activation of gp130, a signaltransducing receptor component for interleukin-6-related cytokines, causes myocardial hypertrophy in mice. Proc Natl Acad Sci USA 1995;92:4862-4866.

30 Blake P, Hasegawa Y, Khosla MC, FouadTarazi F, Sakura N, Paganini EP: Isolation of 'myocardial depressant factor(s)' from the ultrafiltrate of heart failure patients with acute renal failure. ASAIO J 1996;42:M911M915.

-31 Edmunds NJ, Lal H, Woodward B: Effects of tumour necrosis factor- $\alpha$ on left ventricular function in the rat isolated perfused heart: possible mechanisms for a decline in cardiac function. Br J Pharmacol 1999;126:189-196.

32 Miyano H, Shishido T, Kawada T, et al: Acute effect of tumor necrosis factor- $\alpha$ is minimal on mechanics but significant on energetics in blood-perfused canine left ventricles. Crit Care Med 1999;27:168-176.

33 Rauchhaus M, Doehner W, Francis DP, et al: Plasma cytokine parameters and mortality in patients with chronic heart failure. Circulation 2000;102:3060-3067.

-34 Anker SD, Coats AJ: How to RECOVER from RENAISSANCE? The significance of the results of RECOVER, RENAISSANCE, RENEWAL and ATTACH. Int J Cardiol 2002;86:123-130.
35 Wen X, Peng Z, Li Y, et al: One dose of cyclosporine $\mathrm{A}$ is protective at initiation of folic acid-induced acute kidney injury in mice. Nephrol Dial Transplant 2012;27: 3100-3109.

36 Gho BC, Schoemaker RG, van den Doel MA, Duncker DJ, Verdouw PD: Myocardial protection by brief ischemia in noncardiac tissue. Circulation 1996;94:2193-2200.

37 Verdouw PD, Gho BC, Koning MM, Schoemaker RG, Duncker DJ: Cardioprotection by ischemic and nonischemic myocardial stress and ischemia in remote organs. Implications for the concept of ischemic preconditioning. Ann NY Acad Sci 1996;793:27-42.

38 Kobuchi S, Tanaka R, Shintani T, et al: Mechanisms underlying the renoprotective effect of GABA against ischemia/reperfusioninduced renal injury in rats. J Pharmacol Exp Ther 2011;338:767-774.

39 Li C, Zhang D, Han S, et al: Synthesis, electronic properties, and applications of indium oxide nanowires. Ann NY Acad Sci 2003;1006:104-121.

40 Singh K, Xiao L, Remondino A, Sawyer DB, Colucci WS: Adrenergic regulation of cardiac myocyte apoptosis. J Cell Physiol 2001;189: 257-265.

41 Kim S, Iwao H: Molecular and cellular mechanisms of angiotensin II-mediated cardiovascular and renal diseases. Pharmacol Rev 2000 52:11-34.

42 Kajstura J, Cigola E, Malhotra A, et al: Angiotensin II induces apoptosis of adult ventricular myocytes in vitro. J Mol Cell Cardiol 1997;29:859-870.

43 Kawano H, Do YS, Kawano Y, et al: Angiotensin II has multiple profibrotic effects in human cardiac fibroblasts. Circulation 2000; 101:1130-1137.

44 Desai KV, Laine GA, Stewart RH, et al: Mechanics of the left ventricular myocardial interstitium: effects of acute and chronic myocardial edema. Am J Physiol Heart Circ Physiol 2008;294:H2428-H2434.

45 Nath KA, Grande JP, Croatt AJ, et al: Transgenic sickle mice are markedly sensitive to renal ischemia-reperfusion injury. Am J Pathol 2005;166:963-972.

46 Robinson SC, Bowmer CJ, Yates MS: Cardiac function in rats with acute renal failure. J Pharm Pharmacol 1992;44:1007-1014. 
47 Kelly KJ, Meehan SM, Colvin RB, Williams WW, Bonventre JV: Protection from toxicant-mediated renal injury in the rat with anti-CD54 antibody. Kidney International 1999;56:922-931.

48 Wencker D, Chandra M, Nguyen K, et al: A mechanistic role for cardiac myocyte apoptosis in heart failure. J Clin Invest 2003;111: 1497-1504.

49 Le NT, Richardson DR: The role of iron in cell cycle progression and the proliferation of neoplastic cells. Biochim Biophys Acta 2002; 1603:31-46.

-50 Liu YH, D’Ambrosio M, Liao TD, et al: $\mathrm{N}$-acetyl-seryl-aspartyl-lysyl-proline prevents cardiac remodeling and dysfunction induced by galectin-3, a mammalian adhesion/ growth-regulatory lectin. Am J Physiol Heart Circ Physiol 2009;296:H404-H412.

51 Wiedemann HP, Wheeler AP, Bernard GR, et al: Comparison of two fluid-management strategies in acute lung injury. N Engl J Med 2006;354:2564-2575.

52 Arikan AA, Zappitelli M, Goldstein SL, Naipaul A, Jefferson LS, Loftis LL: Fluid overload is associated with impaired oxygenation and morbidity in critically ill children. Pediatr Crit Care Med 2012;13:253-258.

53 Bouchard J, Soroko SB, Chertow GM, et al: Fluid accumulation, survival and recovery of kidney function in critically ill patients with acute kidney injury. Kidney Int 2009;76:422427.

54 Payen D, de Pont AC, Sakr Y, Spies C, Reinhart K, Vincent JL: A positive fluid balance is associated with a worse outcome in patients with acute renal failure. Crit Care 2008; 12:R74

55 Grams ME, Estrella MM, Coresh J, Brower RG, Liu KD: Fluid balance, diuretic use, and mortality in acute kidney injury. Clin J Am Soc Nephrol 2011;6:966-973.

56 Bellomo R, Cass A, Cole L, et al, RENAL Replacement Therapy Study Investigators: An observational study fluid balance and patient outcomes in the Randomized Evaluation of Normal vs. Augmented Level of Replacement Therapy trial. Crit Care Med 2012;40:17531760.

57 Ip JE, Cheung JW, Park W: Temporal associations between thoracic volume overload and malignant ventricular arrhythmias: a study of intrathoracic impedance. J Cardiovasc Electrophysiol 2011;22:293-299.
58 Davis KL, Laine GA, Geissler HJ: Effects of myocardial edema on the development of myocardial interstitial fibrosis. Microcirculation 2000;7:269-280.

59 Nazar A, Guevara M, Sitges M, et al: LEFT ventricular function assessed by echocardiography in cirrhosis: relationship to systemic hemodynamics and renal dysfunction. J Hepatol 2013;58:51-57.

60 Legrand M, Darmon M, Joannidis M, Payen D: Management of renal replacement therapy in ICU patients: an international survey. Intensive Care Med 2013;39:101-108.

61 Bellomo R, Cass A, Cole L, et al: Intensity of continuous renal-replacement therapy in critically ill patients. N Engl J Med 2009;361: 1627-1638.

62 Nimmo AJ, Than N, Orchard CH, Whitaker EM: The effect of acidosis on $\beta$-adrenergic receptors in ferret cardiac muscle. Exp Physiol 1993;78:95-103.

63 Bagshaw SM, Wald R, Barton J, et al: Clinical factors associated with initiation of renal replacement therapy in critically ill patients with acute kidney injury - a prospective multicenter observational study. J Crit Care 2012;27:268-275.

64 Palevsky PM, Zhang JH, O’Connor TZ, et al: Intensity of renal support in critically ill patients with acute kidney injury. N Engl J Med 2008;359:7-20.

-65 Schwenger V, Weigand MA, Hoffmann O, et al: Sustained low efficiency dialysis using a single-pass batch system in acute kidney injury - a randomized interventional trial: the REnal Replacement Therapy Study in Intensive Care Unit PatiEnts. Crit Care 2012; 16:R140.

66 Zazzo JF, Troche G, Ruel P, Maintenant J: High incidence of hypophosphatemia in surgical intensive care patients: efficacy of phosphorus therapy on myocardial function. Intensive Care Med 1995;21:826-831.

67 Demirjian S, Teo BW, Guzman JA, et al: Hypophosphatemia during continuous hemodialysis is associated with prolonged respiratory failure in patients with acute kidney injury. Nephrol Dial Transplant 2011; 26:3508-3514.

68 Duranton F, Cohen G, De Smet R, et al: Normal and pathologic concentrations of uremic toxins. J Am Soc Nephrol 2012;23: $1258-1270$. 
69 Neirynck N, Vanholder R, Schepers E, Eloot S, Pletinck A, Glorieux G: An update on uremic toxins. Int Urol Nephrol 2013;45: 139-150.

70 Littmann L, Monroe MH, Taylor L 3rd, Brearley WD Jr: The hyperkalemic Brugada sign. J Electrocardiol 2007;40:53-59.

71 Marques JS, Diogo AN: Dead man walking: an extreme case of sinusoidal wave pattern in severe hyperkalemia. J Am Coll Cardiol 2012; 59:2118.

72 Shannon TR, Pogwizd SM, Bers DM: Elevated sarcoplasmic reticulum $\mathrm{Ca}^{2+}$ leak in intact ventricular myocytes from rabbits in heart failure. Circ Res 2003;93:592-594.

73 Schulz R, Panas DL, Catena R, Moncada S, Olley PM, Lopaschuk GD: The role of nitric oxide in cardiac depression induced by interleukin- $1 \beta$ and tumour necrosis factor- $\alpha$. Br J Pharmacol 1995;114:27-34.

-74 Tune JD, Gorman MW, Feigl EO: Matching coronary blood flow to myocardial oxygen consumption. J Appl Physiol 2004;97:404415.

75 Ronco C, Cruz D, Noland BW: Neutrophil gelatinase-associated lipocalin curve and neutrophil gelatinase-associated lipocalin extended-range assay: a new biomarker approach in the early diagnosis of acute kidney injury and cardio-renal syndrome. Seminars in nephrology 2012;32:121-128.

76 Mishra J, Ma Q, Prada A, et al: Identification of neutrophil gelatinase-associated lipocalin as a novel early urinary biomarker for ischemic renal injury. J Am Soc Nephrol 2003;14:2534-2543.

-77 Haase M, Haase-Fielitz A, Bellomo R, Mertens PR: Neutrophil gelatinase-associated lipocalin as a marker of acute renal disease. Curr Opin Hematol 2010.

78 Schmidt-Ott KM, Mori K, Li JY, et al: Dual action of neutrophil gelatinase-associated lipocalin. J Am Soc Nephrol 2007;18:407413.
79 Cruz DN, Gaiao S, Maisel A, Ronco C, Devarajan P: Neutrophil gelatinaseassociated lipocalin as a biomarker of cardiovascular disease: a systematic review. Clin Chem Lab Medicine 2012;50:1533-1545

-80 Yndestad A, Landro L, Ueland T, et al: Increased systemic and myocardial expression of neutrophil gelatinaseassociated lipocalin in clinical and experimental heart failure. Eur Heart J 2009; 30:1229-1236.

81 Ding L, Hanawa H, Ota Y, et al: Lipocalin-2/ neutrophil gelatinase- $\mathrm{B}$ associated lipocalin is strongly induced in hearts of rats with autoimmune myocarditis and in human myocarditis. Circ J 2010;74:523-530.

82 Carlsson AC, Larsson A, HelmerssonKarlqvist J, et al: Urinary kidney injury molecule 1 and incidence of heart failure in elderly men. Eur J Heart Fail 2012 (E-pub ahead of print).

83 Scheuer J, Stezoski W: The effects of uremic compounds on cardiac function and metabolism. J Mol Cell Cardiol 1973;5:287300.

84 James MT, Ghali WA, Knudtson ML, et al: Associations between acute kidney injury and cardiovascular and renal outcomes after coronary angiography. Circulation 2011;123: 409-416.

85 Selby NM, Kolhe NV, McIntyre CW, et al: Defining the cause of death in hospitalised patients with acute kidney injury. PLoS One 2012;7:e48580.

86 Thakar CV, Christianson A, Himmelfarb J, Leonard AC: Acute kidney injury episodes and chronic kidney disease risk in diabetes mellitus. Clin J Am Soc Nephrol 2011;6: 2567-2572.

87 James MT, Hemmelgarn BR, Wiebe N, et al: Glomerular filtration rate, proteinuria, and the incidence and consequences of acute kidney injury: a cohort study. Lancet 2010; 376:2096-2103.

88 Acute Dialysis Quality Initiative (ADQI). www.ADQI.org (accessed January 10, 2013).

\author{
Dr. Sean M. Bagshaw \\ Division of Critical Care Medicine, University of Alberta Hospital \\ 3C1.12 Walter C. Mackenzie Centre \\ 8440-122 Street, Edmonton, AB T6G2B7 (Canada) \\ E-Mail bagshaw@ualberta.ca
}

